Homology, Homotopy and Applications, vol.7(2), 2005, pp.179-187

\title{
HOMOTOPY THEORIES OF ALGEBRAS OVER OPERADS
}

\author{
V. A. SMIRNOV \\ (communicated by Tom Lada)
}

\begin{abstract}
Homotopy theories over operads are defined. The corresponding spectral sequences for the homotopy groups are constructed. The calculations of the spectral sequences of the homotopy groups over the " $n$-dimensional little cubes" operads are produced.
\end{abstract}

There are two classical homotopy theories: the homotopy theory of topological spaces (the problem of calculating the homotopy groups of spheres is one of the most difficult problems of algebraic topology); the rational homotopy theory (the problem of calculating the homotopy groups of spheres is very simple).

In [1] it was shown that the rational homotopy theory of 1-connected topological spaces is equivalent to the homotopy theory of 1-connected commutative $D G A$ algebras. In [2], [3] it was shown that the singular chain complex $C_{*}(\mathcal{X})$ (cochain complex $C^{*}(\mathcal{X})$ ) of a topological space $\mathcal{X}$ possesses the structure of an $E_{\infty}$-coalgebra ( $E_{\infty}$-algebra), and the homotopy theory of 1-connected topological spaces is equivalent to the homotopy theory of 1-connected $E_{\infty}$-coalgebras ( $E_{\infty}$-algebras).

Here we consider the homotopy theories of algebras over operads and in particular over the " $n$-dimensional little cubes" operads $E_{n}, 1 \leqslant n \leqslant \infty,[4]$. The ground ring will be assumed to be a field. We construct the spectral sequences for these homotopy theories and try to calculate the corresponding homotopy groups.

Recall that a family $\mathcal{E}=\{\mathcal{E}(j)\}_{j} \geqslant 1$ of chain complexes $\mathcal{E}(j)$ acted upon by the symmetric groups $\Sigma_{j}$ is called an operad if there are given operaions

$$
\gamma: \mathcal{E}(k) \otimes \mathcal{E}\left(j_{1}\right) \otimes \cdots \otimes \mathcal{E}\left(j_{k}\right) \rightarrow \mathcal{E}\left(j_{1}+\cdots+j_{k}\right),
$$

which are compatible with the actions of the symmetric groups and satisfy some associativity relations $[\mathbf{2}]$.

A chain complex $X$ is called an algebra (coalgebra) over an operad $\mathcal{E}$ or simply $\mathcal{E}$-algebra $(\mathcal{E}$-coalgebra) if there is given a family of mappings

$$
\mu(j): \mathcal{E}(j) \otimes X^{\otimes j} \rightarrow X, \quad\left(\tau(j): X \rightarrow \operatorname{Hom}\left(\mathcal{E}(j) ; X^{\otimes j}\right),\right.
$$

which are compatible with the actions of the symmetric groups and satisfy some associativity relation $[\mathbf{2}]$.

Suppoted by the RFBR grant 01-01-00482.

Received August 8, 2003, revised October 31, 2003; published on April 22, 2005.

2000 Mathematics Subject Classification: 55P15, 55S20.

Key words and phrases: Operads, homotopy theories.

(C) 2005, V. A. Smirnov. Permission to copy for private use granted. 
Denote the sum

$$
\sum_{j} \mathcal{E}(j) \otimes_{\Sigma_{j}} X^{\otimes j}
$$

by $\mathcal{E}(X)$. The correspondence $X \longmapsto \mathcal{E}(X)$ determines the functor in the categoty of chain complexes and an operad structure determines a natural transformation $\gamma: \mathcal{E} \circ \mathcal{E} \rightarrow \mathcal{E}$ of functors satisfying the associativity relation. It means that this functor is a monad in the category of chain complexes [3].

If $X$ is an algebra over an operad $\mathcal{E}$ then there will be a chain mapping $\mu: \mathcal{E}(X) \rightarrow$ $X$ and hence $X$ will be an algebra over the monad $\mathcal{E}$.

If one wants to consider unitial algebras, the sum in the definition of $\mathcal{E}(X)$ must be modded out by the unit relation [4].

Dually denote

$$
\overline{\mathcal{E}}(X)=\prod_{j} \operatorname{Hom}_{\Sigma_{j}}\left(\mathcal{E}(j) ; X^{\otimes j}\right) .
$$

Then under suitable assumptions (for example if $\mathcal{E}$ is finitely generated) the correspondence $X \longmapsto \overline{\mathcal{E}}(X)$ determines the comonad in the category of chain complexes. $\overline{\mathcal{E}}$.

If $X$ is a coalgebra over an operad $\mathcal{E}$ then it will be a coalgebra over the comonad

Operads and algebras over operads may be considered in the category of topological spaces (in this case we need instead of the tensor products $\otimes$ in the definition of the operation $\gamma$, the usual product $\times$ ) or other symmetric monoidal categories [3].

Consider some examples of operads and algebras (coalgebras) over operads.

1. An operad $E_{0}=\left\{E_{0}(j)\right\}$, where $E_{0}(j)$ is the free module with one zero dimensional generator $e(j)$ and trivial action of the symmetric group $\Sigma_{j}$. So $E_{0}(j) \cong$ $R$. The operation $\gamma: E_{0} \times E_{0} \rightarrow E_{0}$ is defined by the formula

$$
\gamma\left(e(k) \otimes e\left(j_{1}\right) \otimes \cdots \otimes e\left(j_{k}\right)\right)=e\left(j_{1}+\cdots+j_{k}\right) .
$$

It is easy to see that so defined, this operation is associative and compatible with the actions of the symmetric groups.

Algebras (coalgebras) over $E_{0}$ are simply commutative and associative algebras (coalgebras).

2. An operad $A=\{A(j)\}$, where $A(j)$ is the free $\Sigma_{j}$-module with one zero dimensional generator $a(j)$. So $A(j) \cong R\left(\Sigma_{j}\right)$. The operation $\gamma: A \times A \rightarrow A$ is defined by the formula

$$
\gamma\left(a(k) \otimes a\left(j_{1}\right) \otimes \cdots \otimes a\left(j_{k}\right)\right)=a\left(j_{1}+\cdots+j_{k}\right) .
$$

It is easy to see that the required relations are satisfied.

Algebras (coalgebras) over $A$ are simply associative algebras (coalgebras).

3. For any chain complex $X$ define operads $\mathcal{E}_{X}, \mathcal{E}^{X}$ by putting

$$
\mathcal{E}_{X}(j)=\operatorname{Hom}\left(X^{\otimes j} ; X\right) ; \quad \mathcal{E}^{X}(j)=\operatorname{Hom}\left(X ; X^{\otimes j}\right) .
$$


The actions of the symmetric groups are determined by the permutations of factors of $X^{\otimes j}$ and operad structures are defined by the formulas

$$
\begin{aligned}
& \gamma_{X}\left(f \otimes g_{1} \otimes \cdots \otimes g_{k}\right)=f \circ\left(g_{1} \otimes \cdots \otimes g_{k}\right), \quad f \in \mathcal{E}_{X}(k), g_{i} \in \mathcal{E}_{X}\left(j_{i}\right) ; \\
& \gamma^{X}\left(f \otimes g_{1} \otimes \cdots \otimes g_{k}\right)=\left(g_{1} \otimes \cdots \otimes g_{k}\right) \circ f, \quad f \in \mathcal{E}^{X}(k), g_{i} \in \mathcal{E}^{X}\left(j_{i}\right) .
\end{aligned}
$$

A chain complex $X$ is an algebra (coalgebra) over an operad $\mathcal{E}$ if and only if there is given an operad mapping $\xi: \mathcal{E} \rightarrow \mathcal{E}_{X}\left(\xi: \mathcal{E} \rightarrow \mathcal{E}^{X}\right)$.

4. For $n \geqslant 0$ denote by $\Delta^{n}$ the normalized chain complex of the standard $n$ dimensional simplex. Then $\Delta^{*}=\left\{\Delta^{n}\right\}$ is the cosimplicial object in the category of chain complexes. Denote the realization of the cosimplisial object $\left(\Delta^{*}\right)^{\otimes j}=$ $\Delta^{*} \otimes \cdots \otimes \Delta^{*}$ as $E^{\Delta}(j)$, i.e.

$$
E^{\Delta}(j)=\operatorname{Hom}\left(\Delta^{*} ;\left(\Delta^{*}\right)^{\otimes j}\right),
$$

where Hom is considered in the category of cosimplicial objects.

So the elements of $E^{\Delta}(j)$ are the sequences $f=\left\{f^{n}\right\}$ of mappings $f^{n}: \Delta^{n} \rightarrow$ $\left(\Delta^{n}\right)^{\otimes j}$ commuting the diagrams

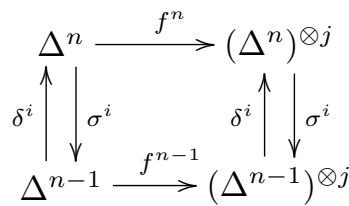

The family $E^{\Delta}=\left\{E^{\Delta}(j)\right\}$ will be the operad for which the actions of the symmetric groups and the operad structure are defined similary to the corresponding structure for the above defined operad $\mathcal{E}^{X}$, where instead of $X$ we take $\Delta^{*}$.

Note that since the complexes $\Delta^{n}$ are acyclic then the operad $E^{\Delta}$ is also acyclic.

In [3] it was shown that on the chain complex $C_{*}(\mathcal{X})$ of a topological space $\mathcal{X}$ there exists a natural $E^{\Delta}$-coalgebra structure. Dually, on the cochain complex $C^{*}(\mathcal{X})$ there exists a natural $E^{\Delta}$-algebra structure.

5. The main examples of topological operads are the little $n$-cube operads $E_{n}$ introduced by Boardman and Vogt [5] and studied by May [4]. Any $n$-fold loop space $\Omega^{n} \mathcal{X}$ is an algebra over the operad $E_{n}$.

There are inclusions $E_{n} \rightarrow E_{n+1}$ and its direct limit denoted as $E_{\infty}$. It is acyclic operad with free actions of the symmetric groups.

Any acyclic operad with free action of the symmetric groups is called $E_{\infty}$-operad. Any algebra (coalgebra) over $E_{\infty}$-operad is called $E_{\infty}$-algebra ( $E_{\infty}$-coalgebra).

6. It is easy to see that if $\mathcal{E}=\{\mathcal{E}(j)\}$ is an operad in the category of topological space then the family $C_{*}(\mathcal{E})=\left\{C_{*}(\mathcal{E}(j))\right\}$ consisting of the corresponding chain complexes will be an operad in the category of chain complexes and if $\mathcal{E}$ is $E_{\infty^{-}}$ operad then $C_{*}(\mathcal{E})$ is $E_{\infty}$-operad.

7. An operad $\mathcal{E}$ is called a Hopf operad if there is given a coassociative operad mapping $\nabla: \mathcal{E} \rightarrow \mathcal{E} \otimes \mathcal{E}$. It is easy to see that $E_{0}, A$ are Hopf operads.

The operad $E^{\Delta}$ is a Hopf operad. The Hopf structure $\nabla: E^{\Delta} \rightarrow E^{\Delta} \otimes E^{\Delta}$ is induced by the diagonal mapping $\Delta^{*} \rightarrow \Delta^{*} \otimes \Delta^{*}$. 
If $\mathcal{E}$ is a toplogical operad then it's singular chain complexes operad $C_{*}(\mathcal{E})$ is a Hopf operad in which the Hopf structure is induced by the coalgebra structures on the $C_{*}(\mathcal{E}(j))$.

8. The singular chain complex $C_{*}(\mathcal{X})$ (cochain complex $C^{*}(\mathcal{X})$ ) is an $E_{\infty}$-coalgebra ( $E_{\infty}$-algebra). Indeed, let $E$ be an $E_{\infty}$-operad. Consider the operad $E^{\Delta} \otimes E$. It is $E_{\infty}$-operad and there is the projection of operads $p: E^{\Delta} \otimes E \rightarrow E^{\Delta}$. Then the composition

$$
E^{\Delta} \otimes E \stackrel{p}{\longrightarrow} E^{\Delta} \stackrel{\xi}{\longrightarrow} \mathcal{E}^{C_{*}(\mathcal{X})} \quad\left(E^{\Delta} \otimes E \stackrel{p}{\longrightarrow} E^{\Delta} \stackrel{\xi}{\longrightarrow} \mathcal{E}_{C^{*}(\mathcal{X})}\right) .
$$

will give on $C_{*}(\mathcal{X})\left(C^{*}(\mathcal{X})\right)$ the structure of $E^{\Delta} \otimes E$-coalgebra $\left(E^{\Delta} \otimes E\right.$-algebra).

Denote the operad $E^{\Delta} \otimes C_{*}\left(E_{n}\right)$ in the category of chain complex simply by $E_{n}$. Then $C_{*}(\mathcal{X})$ may be considered as an $E_{n}$-coalgebra. Dually, $C^{*}(\mathcal{X})$ may be considered as an $E_{n}$-algebra.

We will need the following general properties of algebras (coalgebras) over operads.

Proposition 1. The category of $\mathcal{E}$-algebras (E-coalgebras) over a Hopf operad $\mathcal{E}$ admits tensor products.

Proof. Let $X^{\prime}, X^{\prime \prime}-\mathcal{E}$-algebras, i.e. there are given operad mappings $\xi^{\prime}: \mathcal{E} \rightarrow \mathcal{E}_{X^{\prime}}$, $\xi^{\prime \prime}: \mathcal{E} \rightarrow \mathcal{E}_{X^{\prime \prime}}$. Defing the mapping $\xi: \mathcal{E} \rightarrow \mathcal{E}_{X^{\prime} \otimes X^{\prime \prime}}$ as the composition

$$
\mathcal{E} \stackrel{\nabla}{\longrightarrow} \mathcal{E} \otimes \mathcal{E} \stackrel{\xi^{\prime} \otimes \xi^{\prime \prime}}{\longrightarrow} \mathcal{E}_{X^{\prime}} \otimes \mathcal{E}_{X^{\prime \prime}} \longrightarrow \mathcal{E}_{X^{\prime} \otimes X^{\prime \prime}}
$$

This mapping will give on $X^{\prime} \otimes X^{\prime \prime}$ the desired $\mathcal{E}$-algebra structure.

Proposition 2. If $X_{*}=\left\{X_{n}\right\}$ is a simplicial object in the category of algebras over an operad $\mathcal{E}$ then its realization $\left|X_{*}\right|$ will also be an $\mathcal{E}$-algebra. Dually, if $X^{*}=$ $\left\{X^{n}\right\}$ is a cosimplicial object in the category of coalgebras over an operad $\mathcal{E}$ then its realization $\left|X^{*}\right|$ will be an $\mathcal{E}$-coalgebra.

Proof. Consider a simplicial object $X_{*}=\left\{X_{n}\right\}$ in the category of $\mathcal{E}$-algebras, $\mu_{n}: \mathcal{E}\left(X_{n}\right) \rightarrow X_{n}$, the $\mathcal{E}$-algebra structure on $X_{n}$. The Eilenberg-Zilber mappings

$$
\psi:\left|X_{*}\right| \otimes \cdots \otimes\left|X_{*}\right| \rightarrow\left|X_{*} \otimes \cdots \otimes X_{*}\right|
$$

commute with the actions of the symmetric groups and hence induce mappings

$$
\psi: \mathcal{E}(j) \otimes_{\Sigma_{j}}\left|X_{*}\right|^{\otimes j} \rightarrow\left|\mathcal{E}(j) \otimes_{\Sigma_{j}} X_{*}^{\otimes j}\right| .
$$

These mappings give us the mapping $\psi: \mathcal{E}\left(\left|X_{*}\right|\right) \rightarrow\left|\mathcal{E}\left(X_{*}\right)\right|$ and desired mapping $\mathcal{E}\left(\left|X_{*}\right|\right) \rightarrow\left|X_{*}\right|$ is the composition

$$
\mathcal{E}\left(\left|X_{*}\right|\right) \stackrel{\psi}{\longrightarrow}\left|\mathcal{E}\left(X_{*}\right)\right| \stackrel{\mu_{*}}{\longrightarrow}\left|X_{*}\right| .
$$

Corollary. The realization $B(\mathcal{E}, \mathcal{E}, X)$ of the simplicial resolution

$$
B_{*}(\mathcal{E}, \mathcal{E}, X): \mathcal{E}(X) \longleftarrow \mathcal{E}^{2}(X) \longleftarrow \cdots \longleftarrow \mathcal{E}^{n}(X) \longleftarrow \cdots
$$


over an $\mathcal{E}$-algebra $X$ is an $\mathcal{E}$-algebra with chain equivalence $\eta: B(\mathcal{E}, \mathcal{E}, X) \rightarrow X$. Dually, the realization $F(\mathcal{E}, \mathcal{E}, X)$ of the cosimplicial resolution

$$
F^{*}(\mathcal{E}, \mathcal{E}, X): \overline{\mathcal{E}}(X) \longrightarrow \overline{\mathcal{E}}^{2}(X) \longrightarrow \cdots \longrightarrow \overline{\mathcal{E}}^{n}(X) \longrightarrow \cdots
$$

over an $\mathcal{E}$-coalgebra $X$ is an $\mathcal{E}$-coalgebra with chain equivalence $\xi: X \rightarrow F(\mathcal{E}, \mathcal{E}, X)$.

Pass now to the homotopy theories. Let $\mathcal{E}$ be a Hopf operad for which there is given operad mapping $\mathcal{E} \rightarrow \mathcal{E}^{\Delta}$. It means that the chain complexes $\Delta^{n}$ possess $\mathcal{E}$-coalgebra structures compatible with the coface and codegeneracy operators. In particular, the unit segment $I=\Delta^{1}$ possesses $\mathcal{E}$-coalgebra structure.

Denote $\mathcal{A}_{\mathcal{E}}\left(\mathcal{K}_{\mathcal{E}}\right)$ the category in which objects are $\mathcal{E}$-algebras $(\mathcal{E}$-coalgebras) and morphisms are $\mathcal{E}$-algebra mappings ( $\mathcal{E}$-coalgebra mappings).

In [6] there are given sufficient conditions for the existence of a closed model structure on the category of operads in an arbitrary symmetric monoidal category. In particular chain operads carry a closed model structure.

Here we prove that the category $\mathcal{A}_{\mathcal{E}}\left(\mathcal{K}_{\mathcal{E}}\right)$ possesses a closed model structure [7].

Define a map in $\mathcal{A}_{\mathcal{E}}$ to be a weak equivalence if it induces isomorphism on homology, a fibration if it is surjective and a cofibration if it has the left lifting property with respect to all trivial fibrations.

Theorem 1. The category $\mathcal{A}_{\mathcal{E}}$ is a closed model category.

Proof. As in the case of usual algebras $[\mathbf{7}]$ the only nontrivial part of the theorem to prove is that any map $f: X \rightarrow Y$ of $\mathcal{E}$-algebras may be factored into the composition $f=p \circ i$, where $i$ is a cofibration and $p$ is a trivial fibration.

The idea of the proof repeats the corresponding proof for usual algebras. Namely, let $f: X \rightarrow Y$ be a mapping of $\mathcal{E}$-algebras. Define an $\mathcal{E}$-algebra $\mathcal{E}(X, Y)$, putting $\mathcal{E}(X, Y)=X+\mathcal{E}(Y)$. An $\mathcal{E}$-algebra structure is induced by $\mathcal{E}$-algebra structures on $X$ and $\mathcal{E}(Y)$.

There is a projection $p: \mathcal{E}(X, Y) \rightarrow Y$, induced by the mapping $f: X \rightarrow Y$ and the $\mathcal{E}$-algebra structure $\mu: \mathcal{E}(Y) \rightarrow Y, p(x+y)=f(x)+\mu(y)$. Besides that there are an injection $i: X \rightarrow \mathcal{E}(X, Y)$ and a chain mapping $j: Y \rightarrow \mathcal{E}(X, Y)$ such that $p \circ i=f, p \circ j=I d$. More over $p$ is a fibration and $i$ is a cofibration. However $p$ is not a trivial fibration. To improve this fibration we construct a simplicial resolution $\mathcal{E}_{*}(X, Y)$, putting

$$
\mathcal{E}_{0}(X, Y)=\mathcal{E}(X, Y), \quad \mathcal{E}_{n+1}(X, Y)=\mathcal{E}\left(X, \mathcal{E}_{n}(X, Y)\right) .
$$

The face and degeneracy mappings are defined by the inductive formulas. Namely,

$$
d_{0}=p: \mathcal{E}(X, Y) \rightarrow Y, \quad s_{0}=\mathcal{E}(-, j) \mathcal{E}(X, Y) \rightarrow \mathcal{E}_{1}(X, Y)=\mathcal{E}(X, \mathcal{E}(X, Y)) .
$$

Similary there are defined

$$
\begin{gathered}
d_{0}=p: \mathcal{E}_{n+1}(X, Y)=\mathcal{E}\left(X, \mathcal{E}_{n}(X, Y)\right) \rightarrow \mathcal{E}_{n}(X, Y) \\
s_{0}=\mathcal{E}(-, j): \mathcal{E}_{n}(X, Y) \rightarrow \mathcal{E}_{n+1}(X, Y)=\mathcal{E}\left(X, \mathcal{E}_{n}(X, Y)\right) .
\end{gathered}
$$


Finally, define

$$
\begin{aligned}
& d_{i+1}=\mathcal{E}\left(-, d_{i}\right): \mathcal{E}_{n+1}(X, Y) \rightarrow \mathcal{E}_{n}(X, Y) ; \\
& s_{i+1}=\mathcal{E}\left(-, s_{i}\right): \mathcal{E}_{n}(X, Y) \rightarrow \mathcal{E}_{n+1}(X, Y) .
\end{aligned}
$$

Note that if $X$ is a trivial then $\mathcal{E}_{*}(X, Y)$ is isomorphic to $B_{*}(\mathcal{E}, \mathcal{E}, Y)$.

The realization $\left|\mathcal{E}_{*}(X, Y)\right|$ is an $\mathcal{E}$-algebra which is chain equivalent to $Y$. Moreover there are the surjective mapping $p:\left|\mathcal{E}_{*}(X, Y)\right| \rightarrow Y$ and an injective mapping $i: X \rightarrow\left|\mathcal{E}_{*}(X, Y)\right|$ such that $f=p \circ i$. If $X$ is a trivial we have the isomorphism $\left|\mathcal{E}_{*}(X, Y)\right| \cong B(\mathcal{E}, \mathcal{E}, Y)$.

We prove that the mapping $i: X \rightarrow\left|\mathcal{E}_{*}(X, Y)\right|$ is a cofibration. Let $u: U \rightarrow V$ be a trivial fibration. It means that $u$ is an surjective and induces an isomorphism of homologies. Then there is a chain mapping $v: V \rightarrow U$ and a chain homotopy $w: U \rightarrow U$ such that

$$
u \circ u=I d ; \quad d(w)=v \circ u-I d ; \quad u \circ w=0 ; \quad w \circ v=0 ; \quad w \circ w=0 .
$$

Further, let $g: X \rightarrow U, h:\left|\mathcal{E}_{*}(X, Y)\right| \rightarrow V$ be $\mathcal{E}$-algebra mappings commuting the diagram

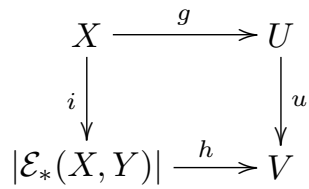

We need to construct an $\mathcal{E}$-algebra mapping $\widetilde{h}:\left|\mathcal{E}_{*}(X, Y)\right| \rightarrow U$ preserving commutativity of the diagram.

It is easy to see that giving an $\mathcal{E}$-algebra mapping $\widetilde{h}:\left|\mathcal{E}_{*}(X, Y)\right| \rightarrow U$ is equivalent to giving a family of $\mathcal{E}$-algebra mappings $h^{n}: \mathcal{E}_{n}(X, Y) \rightarrow H o m\left(\Delta^{n} ; U\right)$ such that the following diagrams are commutative

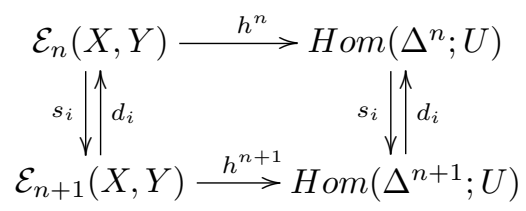

Note that to giving $\mathcal{E}$-algebra mapings $h^{n}: \mathcal{E}_{n}(X, Y) \rightarrow \operatorname{Hom}\left(\Delta^{n} ; U\right)$ is equivalent to giving a mapping on $X$ (determined by $g$ ) and a chain mapping

$$
\bar{h}^{n}: \mathcal{E}_{n-1}(X, Y) \rightarrow \operatorname{Hom}\left(\Delta^{n} ; U\right) .
$$

So we conclude that to give an $\mathcal{E}$-algebra mapping $\widetilde{h}: \mid \mathcal{E}_{*}(X, Y) \rightarrow U$ is equivalent to give a family of chain mappings $\bar{h}^{n}: \mathcal{E}_{n-1}(X, Y) \rightarrow \operatorname{Hom}\left(\Delta^{n} ; U\right)$ such that the corresponding mappings $h^{n}$ are $\mathcal{E}$-algebra mappings commuting the above diagram.

We put $\bar{h}^{0}=v \circ h: Y \rightarrow U$ and $\bar{h}^{n}=w \circ \mu \circ \mathcal{E}\left(g, \bar{h}^{n-1}\right)$. Straight verifications show that the required relations are satisfied.

Corollary. For any $\mathcal{E}$-algebra $Y$ the $\mathcal{E}$-algebra $B(\mathcal{E}, \mathcal{E}, Y)$ is a cofibrant object in the category $\mathcal{A}_{\mathcal{E}}$. 
It follows from the fact that for trivial $X$ there is the isomorphism $\left|\mathcal{E}_{*}(X, Y)\right| \cong$ $B(\mathcal{E}, \mathcal{E}, Y)$.

Dually consider the category $\mathcal{K}_{\mathcal{E}}$. Define a map of this category to be a weak equivalences if it induces the isomorphism on homology a cofibration if it is injective and fibration if it has the right lifting property with respect to all trivial cofibrations.

Theorem (1'). The category $\mathcal{K}_{\mathcal{E}}$ is a closed model category.

Denote by $\operatorname{Ho}_{\mathcal{E}}$ the localization of the category $\mathcal{K}_{\mathcal{E}}$ with respect to the class of weak equivalences, i.e. morphisms induce the isomorphisms of homologies.

For an $\mathcal{E}$-coalgebra $X$ the tensor product $X \otimes \Delta^{1}$ will be a cylinder object, and $\mathcal{E}$ coalgebra mappings $f_{0}, f_{1}: X \rightarrow Y$ will be left homotopic if there exists a mapping $h: X \otimes \Delta^{1} \rightarrow Y$ such that $h \circ \delta^{0}=f_{0}, h \circ \delta^{1}=f_{1}$.

Let $\widetilde{\mathcal{K}}_{\mathcal{E}}$ denote the category, whose objects are $\mathcal{E}$-coalgebras and morphisms $f: X \rightarrow Y$ are $\mathcal{E}$-coalgebra mappings $\tilde{f}: X \rightarrow F(\mathcal{E}, \mathcal{E}, Y)$.

Denote by $\pi \mathcal{K}_{\mathcal{E}}$ the category whose objects are $\mathcal{E}$-coalgebras and morphisms are the homotopy classes of morphisms in $\widetilde{\mathcal{K}}_{\mathcal{E}}$. From general homotopy theory $[\boldsymbol{7}]$ it follows

Theorem 2. There is an equivalence of categories

$$
H o \mathcal{K}_{\mathcal{E}} \cong \pi \mathcal{K}_{\mathcal{E}}
$$

Dually, for $\mathcal{E}$-algebras we have

Theorem (2'). There is an equivalence of categories

$$
H o \mathcal{A}_{\mathcal{E}} \cong \pi \mathcal{A}_{\mathcal{E}} .
$$

Consider now the problem of calculating the homotopy groups of $\mathcal{E}$-coalgebras. $\mathcal{E}$ will be assumed to satisfy some suitable assumptions, for example $\mathcal{E}$ is finitely generated.

Since the chain complexes $\Delta^{n}$ of the standard $n$-dimensional simplexes are $\mathcal{E}$ coalgebras, the chain complexes $S^{n}$ of the $n$-dimensional spheres will be $\mathcal{E}$-coalgebras. Define the homotopy groups $\pi_{n}^{\mathcal{E}}(X)$ of an $\mathcal{E}$-coalgebra $X$ by putting $\pi_{n}^{\mathcal{E}}(X)=$ $\left[S^{n} ; F(\mathcal{E}, \mathcal{E}, X)\right]$, the set of homotopy classes of $\mathcal{E}$-coalgebra mappings $f: S^{n} \rightarrow$ $F(\mathcal{E}, \mathcal{E}, X)$.

Theorem 3. For any $\mathcal{E}$-coalgebra $X$ there is the spectral sequence of the homotopy groups $\pi_{*}^{\mathcal{E}}(X)$ in which the $E^{1}$ term is isomorphic to the cobar construction $F\left(\mathcal{E}_{*}, X_{*}\right)$, where $\mathcal{E}_{*}, X_{*}$ denotes the homologies of $\mathcal{E}$ and $X$ correspondingly.

Proof. Consider the filtration

$$
F(\mathcal{E}, \mathcal{E}, X) \supset F^{1}(\mathcal{E}, \mathcal{E}, X) \supset \cdots \supset F^{m}(\mathcal{E}, \mathcal{E}, X) \supset \ldots,
$$

where $F^{m}(\mathcal{E}, \mathcal{E}, X): \overline{\mathcal{E}}^{m}(X) \longrightarrow \overline{\mathcal{E}}^{m+1}(X) \longrightarrow \cdots$.

This filtration induces the spectral sequence. Exact sequences

$$
0 \rightarrow F^{m+1}(\mathcal{E}, \mathcal{E}, X) \rightarrow F^{m}(\mathcal{E}, \mathcal{E}, X) \rightarrow \overline{\mathcal{E}}^{m+1}(X) \rightarrow 0
$$


induce the isomorphisms

$$
E_{n, m}^{1}=\left[S^{n}, \overline{\mathcal{E}}^{m+1}(X)\right] \cong H_{n}\left(\overline{\mathcal{E}}^{m}(X)\right)
$$

and hence the isomorphism $E^{1} \cong F\left(\mathcal{E}_{*}, X_{*}\right)$.

If $S^{n}$ is a trivial $\mathcal{E}$-coalgebra then the differentials of the spectral sequence are determined only by the differentials of the cobar construction $F(\mathcal{E}, X)$ and thus we have

Theorem 4. If $S^{n}$ is a trivial $\mathcal{E}$-coalgebra then for any $\mathcal{E}$-coalgebra $X$ there is an isomorphism

$$
\pi_{n}^{\mathcal{E}}(X) \cong H_{n}(F(\mathcal{E}, X))
$$

Now let $E_{n}$ be the little $n$-cube operad. Note that if $m \geqslant n$ then the homology of $\bar{E}_{n}\left(S^{m}\right)$ is trivial up to the dimension $2 m-n+1>m$. From here it follows that $S^{m}$ has trivial $E_{n}$-coalgebra structure and hence we have

Theorem 5. If $\mathcal{X}$ is a topological space, $m \geqslant n$ then there is an isomorphism

$$
\pi_{m}^{E_{n}}(\mathcal{X}) \cong H_{m}\left(F\left(E_{n}, C_{*}(\mathcal{X})\right)\right) .
$$

The $E^{1}$-term of the spectral sequence is expressed through the Dyer-Lashof algebra $[\mathbf{8}],[\mathbf{9}]$ and the result is the following

Theorem 6. The $E^{1}$-term of the spectral sequence of the homotopy groups $\pi_{*}^{E_{n}}(\mathcal{X})$ of a topological space $\mathcal{X}$ is isomorphic to the module $S^{n} T_{s} R_{n-1} L_{n-1} S^{-n} H_{*}(\mathcal{X})$, where $T_{s}$ is the free commutative algebra, $R_{n-1}$ is the submodule of the Dyer-Lashof algebra generated by allowable sequence of excess less then $n, L_{n-1}$ is the free $(n-1)$ Lie algebra.

If $\mathcal{X}-n$-connected topological space then the homology of the cobar construction $F\left(E_{n}, C_{*}(\mathcal{X})\right)$ is isomorphic to the $n$-fold suspension over the homology of iterated loop space $\Omega^{n} \mathcal{X}[\mathbf{9}]$. Hence we have

Theorem 7. If $\mathcal{X}$ is an $n$-connected topological space then there is the isomorphism

$$
\pi_{*}^{E_{n}}(X) \cong S^{n} H_{*}\left(\Omega^{n} \mathcal{X}\right) .
$$

\section{References}

[1] Quillen D., Rational homotopy theory. Ann. of Math. 90(1969), N2, 205295.

[2] Smirnov V.A., On the cochain complex of topological spaces. Mat. Sb. (Russia), 115(1981), 146-158.

[3] Smirnov V.A., Homotopy theory of coalgebras. Izv. Ac. Nauk (Russia) 49 (1985), 1302-1321.

[4] May J.P., The geometry of iterated loop spaces. Lect. Notes in Math. 271(1972). 
[5] Boardman J.M., Vogt R.M., Homotopy invariant algebraic structures on topological spaces. Lect. Notes in Math. 347(1973).

[6] Berger C., Moerdijk I., Axiomatic homotopy theory for operads. arXiv:math. AT/0206094 v2 4 Apr 2003.

[7] Quillen D., Homotopical algebra. Lect. Notes in Math. 43(1967).

[8] Dyer E., Lashof R., Homology of iterated loop spaces. Amer. J. Math. 84(1962), 35-88.

[9] Smirnov V.A., The homology of iterated loop spaces. Forum Mathematicum 14(2002), 345-381.

This article may be accessed via WWW at http://www.rmi.acnet.ge/hha/ or by anonymous ftp at

ftp://ftp.rmi.acnet.ge/pub/hha/volumes/2005/n2a10/v7n2a10.(dvi,ps,pdf)

V. A. Smirnov v.smirnov@ru.net

Moscow State Pedagogical University,

Krasnoprudnaya st. 14,

Moscow, 107140,

Russia 